\title{
SUCCESSIVE COEFFICIENTS OF STARLIKE FUNCTIONS
}

\section{YUK LEUNG}

Let $S$ denote the class of functions

$$
f(z)=z+a_{2} z^{2}+\ldots
$$

which are analytic and univalent in the unit disk $|z|<1$. Also, let $S^{*}$ denote the subclass of $S$ consisting of functions which map the unit disk onto domains which are starlike with respect to the origin. The purpose of this paper is to prove a conjecture made by Pommerenke [3, Problem 3.5]; namely, ||$a_{n+1}|-| a_{n}|| \leqslant 1$ for all $f \in S^{*}$.

THEOREM. For every $f \in S^{*}$,

$$
|| a_{n+1}|-| a_{n}|| \leqslant 1, \quad n=1,2,3, \ldots
$$

Equality occurs for fixed $n$ only for the functions

$$
\frac{z}{(1-\gamma z)(1-\zeta z)}
$$

for some $\gamma$ and $\zeta$ with $|\gamma|=|\zeta|=1$.

CoRollary 1. For every $f \in S^{*}$,

$$
\left|a_{n}\right| \leqslant n, \quad n=2,3, \ldots
$$

COROLLARY 2. For every odd function $f \in S^{*}$,

$$
\left|a_{2 n+1}\right| \leqslant 1, \quad n=1,2, \ldots
$$

The first corollary is well known and the second is due to Privalov [5].

The proof of the theorem depends on a lemma due to MacGregor [2], concerning the class $P$ of functions

$$
p(z)=1+\sum_{n=1}^{\infty} p_{n} z^{n}
$$

analytic and of positive real part in $|z|<1$.

LEMMA. Let $p \in P$, and let $\lambda_{n} \geqslant 0$. If $q(z)=\sum_{n=1}^{\infty} \lambda_{n} p_{n} z^{n}$ is analytic in $|z|<1$ and $\operatorname{Re}\{q(z)\} \leqslant M$ for some positive $M$, then $\sum_{n=1}^{\infty} \lambda_{n}\left|p_{n}\right|^{2} \leqslant 2 M$.

Received 10 August, 1977.

[BULl. LoNDON MATH. Soc., 10 (1978), 193-196] 
Proof of the lemma. Let $p_{n}=c_{n}+i d_{n}$ and

$$
\begin{gathered}
u(r, \theta)=\operatorname{Re}\left\{p\left(r e^{i \theta}\right)\right\}=1+\sum_{n=1}^{\infty}\left(c_{n} \cos n \theta-d_{n} \sin n \theta\right) r^{n} \\
v(r, \theta)=\operatorname{Re}\left\{q\left(r e^{i \theta}\right)\right\}=\sum_{n=1}^{\infty} \lambda_{n}\left(c_{n} \cos n \theta-d_{n} \sin n \theta\right) r^{n} \\
\int_{0}^{2 \pi} u(r, \theta) v(r, \theta) d \theta=\pi \sum_{n=1}^{\infty} \lambda_{n}\left(c_{n}{ }^{2}+d_{n}{ }^{2}\right) r^{2 n}=\pi \sum_{n=1}^{\infty} \lambda_{n}\left|p_{n}\right|^{2} r^{2 n}
\end{gathered}
$$

Since $u \geqslant 0$ and $v \leqslant M$,

$$
\int_{0}^{2 \pi} u(r, \theta) v(r, \theta) d \theta \leqslant M \int_{0}^{2 \pi} u(r, \theta) d \theta=2 M .
$$

Thus $\sum_{n=1}^{\infty} \lambda_{n}\left|p_{n}\right|^{2} r^{2 n} \leqslant 2 M$. Letting $r \rightarrow 1$, we have the lemma.

COROLlary. For every $p \in P$ and every positive integer $n$, there is a $\zeta$ with $|\zeta|=1$ such that

$$
\sum_{k=1}^{n} \frac{1}{k}\left|p_{k}-\zeta^{k}\right|^{2} \leqslant \sum_{k=1}^{n} \frac{1}{k}
$$

Proof. Apply the lemma with

This gives

$$
q(z)=\sum_{k=1}^{n} \frac{1}{k} p_{k} z^{k}
$$

$$
\begin{aligned}
\sum_{k=1}^{n} \frac{1}{k}\left|p_{k}-\zeta^{k}\right|^{2} & =\sum_{k=1}^{n} \frac{1}{k}\left|p_{k}\right|^{2}-2 \operatorname{Re}\{q(\zeta)\}+\sum_{k=1}^{n} \frac{1}{k} \\
& \leqslant 2 M-2 \operatorname{Re}\{q(\zeta)\}+\sum_{k=1}^{n} \frac{1}{k} .
\end{aligned}
$$

Choosing $\zeta$ with $|\zeta|=1$ so that $\operatorname{Re}\{q(\bar{\zeta})\}=M=\operatorname{Max} \operatorname{Re}\{q(z)\}$, we obtain the result.

Proof of Theorem. If $f \in S^{*}$, then it is.well known that

$$
\frac{z f^{\prime}(z)}{f(z)}=p(z)
$$

for some $p \in P$. Simple integration gives

$$
\log \frac{f(z)}{z}=\int_{0}^{z} \frac{p(t)-1}{t} d t=\sum_{k=1}^{\infty} \frac{1}{k} p_{k} z^{k}
$$


For $|\zeta|=1$,

$$
\log \left\{(1-\zeta z) \frac{f(z)}{z}\right\}=\sum_{k=1}^{\infty} \alpha_{k} z^{k}
$$

where

$$
\alpha_{k}=\frac{1}{k}\left(p_{k}-\zeta^{k}\right)
$$

On the other hand,

$$
(1-\zeta z) \frac{f(z)}{z}=\sum_{k=0}^{\infty} \beta_{k} z^{k}
$$

where

Because

$$
\beta_{k}=a_{k+1}-\zeta a_{k}
$$

$$
\sum_{k=0}^{\infty} \beta_{k} z^{k}=\exp \left\{\sum_{k=1}^{\infty} \alpha_{k} z^{k}\right\},
$$

we may apply one of the Lebedev-Milin inequalities (see [4], Lemma 3.4) to get

$$
\left|\beta_{n}\right|^{2} \leqslant \exp \left\{\sum_{k=1}^{n} k\left|\alpha_{k}\right|^{2}-\sum_{k=1}^{n} \frac{1}{k}\right\}
$$

with equality only for $\alpha_{k}=\left(\gamma^{k} / k\right), k=1, \ldots, n$, with $|\gamma|=1$. In other words, we have

$$
\left|a_{n+1}-\zeta a_{n}\right|^{2} \leqslant \exp \left\{\sum_{k=1}^{n} \frac{1}{k}\left|p_{k}-\zeta^{k}\right|^{2}-\sum_{k=1}^{n} \frac{1}{k}\right\} .
$$

By the corollary to the lemma, we can pick some $\zeta$ with $|\zeta|=1$ to make the exponent nonpositive. Hence $\left|a_{n+1}-\zeta a_{n}\right| \leqslant 1$. Because ||$a_{n+1}|-| a_{n}|| \leqslant\left|a_{n+1}-\zeta a_{n}\right|$ for all $|\zeta|=1$, this completes the proof of the inequality.

If ||$a_{n+1}|-| a_{n}||=1$, then equality occurs in (2) for some $\zeta$. Hence we have

$$
\sum_{k=0}^{\infty} \beta_{k} z^{k}=\exp \left\{\sum_{k=1}^{n} \frac{\gamma^{k}}{k} z^{k}+O\left(z^{n+1}\right)\right\} .
$$

Comparing the Taylor coefficients of both sides, we get

or

$$
\beta_{k}=\gamma^{k} \text { for } k=1,2 \ldots, n,
$$

$$
a_{k+1}=\zeta a_{k}+\gamma^{k}, \quad k=1,2, \ldots, n .
$$

A simple inductive argument gives

$$
a_{k+1}=\frac{\gamma^{k+1}-\zeta^{k+1}}{\gamma-\zeta}, \quad k=1,2, \ldots, n .
$$

Hence

$$
\left|a_{k+1}\right|=\left|\frac{1-e^{i(k+1) \theta}}{1-e^{i \theta}}\right|, \quad e^{i \theta}=\zeta / \gamma .
$$


Thus the assumption that ||$a_{n+1}|-| a_{n}||=1$ leads to

In general, however,

$$
|| 1-e^{i(n+1) \theta}|-| 1-e^{i n \theta}||=\left|1-e^{i \theta}\right| .
$$

$$
|| 1-e^{i(n+1) \theta}|-| 1-e^{i n \theta}|| \leqslant\left|e^{i(n+1) \theta}-e^{i n \theta}\right|=\left|1-e^{i \theta}\right|,
$$

with equality occurring only when the two vectors $1-e^{i(n+1) \theta}$ and $1-e^{i n \theta}$ are collinear. Hence we have three cases to consider: (1) $\theta=0$; (2) $1-e^{i n \theta}=0$; (3) $1-e^{i(n+1) \theta}=0$. In the first case, when $\theta=0$, we get $\zeta=\gamma$ and $\left|a_{2}\right|=|\zeta+\gamma|=2$, therefore, $f(z)$ must be a rotation of the Koebe function $z /(1-z)^{2}$. In the second case, when $1-e^{i n \theta}=0$, we get $a_{n}=0,\left|a_{n+1}\right|=1$. Let $V_{k+1}^{*}$ be the $2 k$-dimensional region composed of all points $\left(a_{2}, a_{3}, \ldots, a_{k+1}\right)$ with $z+\sum_{n=2}^{\infty} a_{n} z^{n}$ belonging to $S^{*}$. The second case tells us that $\left(a_{2}, \ldots, 0, a_{n+1}\right)$ must be on the boundary of $V_{n+1}^{*}$. Otherwise we would have $\left(\rho a_{2}, \ldots, 0, \rho a_{n+1}\right) \in V_{n+1}^{*}$ for some $\rho>1$. This is impossible because we would get ||$\rho a_{n}|-| \rho a_{n+1}||=\rho>1$. Hence by a theorem of Hummel [1, Theorem $1 \|$, we get a unique $\left(a_{2}, \ldots, 0, a_{n+1}, a_{n+2}\right)$ belonging to $V_{n+2}^{*}$ with

$$
a_{n+2}=\frac{\gamma^{n+2}-\zeta^{n+2}}{\gamma-\zeta} \text {. }
$$

We can repeat the above argument to show that $\left(a_{2}, \ldots, 0, a_{n+1}, a_{n+2}\right)$ is on the boundary of $V_{n+2}^{*}$ and obtain, in general,

$$
a_{n+j}=\frac{\gamma^{n+j}-\zeta^{n+j}}{\gamma-\zeta} \text { for all positive } j
$$

Thus, our function in this case has the form $z /((1-\gamma z)(1-\zeta z))$. Similarly, we get the same result for the third case. This completes the proof of the theorem.

The writer wishes to thank Professor Duren for his help.

\section{References}

1. J. A. Hummel, “ The coefficient regions of starlike functions ", Pacific J. Math., 7 (1957), 13811389.

2. T. H. MacGregor, "An inequality concerning analytic functions with a positive real part ", Canad. J. Math., 21 (1969), 1172-1177.

3. Ch. Pommerenke, "Problems aus der Funktionentheorie", Jber. Deutsch. Math-Verein., 73 (1971), 1-5.

4. Ch. Pommerenke, Univalent Functions (Vandenhoeck and Repurecht, Göttingen, 1975).

5. I. I. Privalov, "On functions giving a univalent conformal mapping", Mat. Sb., 31 (1924), 350-365 (in Russian).

Department of Mathematics,

University of Michigan,

Ann Arbor, Michigan 48109. 